\title{
Peripheral Neuropathy in Wild-Type Amyloidosis: The More You Look, the More You Will Find
}

Keywords: Amyloid, Peripheral neuropathy, Transthyretin

doi:10.1017/cjn.2021.1

Can J Neurol Sci. 2021; 48: 597-598

Transthyretin (TTR) amyloidosis is a progressive systemic disorder caused by TTR protein deposition in affected organs, most commonly the heart and the peripheral nerves. ${ }^{1}$ It can arise from mutated TTR (ATTRv; v for variant) or wild-type TTR protein (ATTRwt) deposition. Different phenotypes have been described in ATTRv, depending on the specific genetic mutations. While the most common North American mutation I122V tends to present as pure or predominant cardiomyopathy in late middle age, the V30M mutation, endemic in Japan and Portugal, has been associated with earlier age of onset of somatic and autonomic peripheral nerve dysfunction followed by cardiac conduction defects. However, the same V30M mutation has also been described in non-endemic areas with late onset after the age of 50, more significant cardiomyopathy, large and small-fiber sensory neuropathy with only mild autonomic dysfunction, suggesting that there are other contributing factors to the phenotype, either environmental or genetic. In contrast, ATTRwt was thought to be associated with a more uniform cardiac presentation of hypertrophic heart failure, with relatively preserved systolic function early in the disease. As ATTRwt occurs almost exclusively in elderly men, it has long been referred to as "senile amyloidosis". Over time, the association with carpal tunnel syndrome, ${ }^{2}$ lumbar spinal stenosis, ${ }^{3}$ mild peripheral neuropathy, and most recently skeletal myopathy ${ }^{4}$ have expanded the clinical spectrum of the disorder.

The retrospective cohort study by Russel et al. ${ }^{5}$ in this issue of the journal reports the type and frequency of peripheral nerve complications in ATTRwt. Consecutive ATTRwt cardiomyopathy patients seen in their multidisciplinary amyloid clinic were offered a complete neurologic evaluation through standardized physical and neurophysiologic examinations. Forty-one of 51 patients agreed and completed the assessment. While the study confirmed the high prevalence of carpal tunnel syndrome in this population ( $88 \%$, the majority symptomatic), it also established a much higher frequency of spinal stenosis (37\%) and of polyneuropathy $(>50 \%)$ than previously recognized. More importantly, it described the clinical and electrophysiologic features of the neuropathy (sensory in $12 / 21$, sensorimotor in 9/21, asymptomatic in 9/21) and uncovered ulnar neuropathy as another potential complication of ATTRwt (34\%), albeit often asymptomatic. The authors compared their findings in this population with age-matched controls to assess the relative contributions of ATTRwt versus simple aging. Finally, they also examined the consequence of these evaluations, as almost half $(46 \%)$ led to further diagnostic testing or therapeutic interventions.

Despite its retrospective nature, this study has several strengths. The authors should be commended for the thorough and systematic clinical and electrophysiological assessment that all patients with wtTTR cardiomyopathy underwent. A recent study hinted at this higher prevalence of neuropathy in ATTRwt patients. ${ }^{6}$ However, it was based solely on a chart review of the patient's sensory symptoms in the lower limbs. It would have missed asymptomatic neuropathy patients and could not differentiate between spinal stenosis and polyneuropathy. Another strength of the current study is the detailed methodology used in order to establish a diagnosis of polyneuropathy, as pure electrophysiologic criteria are often less reliable in older patients. The categorization of patients into two groups, with and without comorbidities potentially causing polyneuropathy, was also very helpful in showing a similar rate of polyneuropathy in the two groups, suggesting again the link with ATTRwt.

The main limitations of the study are its retrospective singlecenter cohort design, the lack of blinding of the examining neurologist, the small number of patients, and the lack of autonomic testing, all of which the authors acknowledge. Genetic testing was not performed on all ATTRwt patients; and while the diagnosis of ATTRv was ruled out by mass spectrometry in those without genetic testing, DNA analysis is now recommended in all patients. ${ }^{7}$

The major difficulty remains in establishing a cause-and-effect relationship between polyneuropathy and ATTRwt. The small control group (15 patients) helps, but is insufficient. In particular, control patients were referred for myasthenia gravis assessments, but did not have heart disease. Some studies have suggested that heart failure by itself may be linked with polyneuropathy. ${ }^{8}$ The higher frequency of polyneuropathy in more severe heart failure may be more related to the heart failure than to amyloid deposition.

Despite these shortcomings, this study supports neurologic evaluation in patients with cardiac ATTRwt and starts to build some real-world evidence in favor of a multidisciplinary approach to this systemic disorder. While guidelines often recommend such an approach, there is a dearth of studies supporting this position. The study's follow-up data demonstrating that evaluation often leads to a change in management is potentially important when discussions about the cost of multidisciplinary clinics arise.

As with many good studies, this study's findings lead to other questions, particularly about treatment. The presence of

Received December 23, 2020. Final Revisions Submitted December 30, 2020. Date of Acceptance January 3, 2021. 
significant neuropathy often guides drug choices in ATTRv, but more data is needed about the response of neuropathy symptoms and signs to therapy in ATTRwt. While the mild nature of most of the neuropathies described may suggest that they are unlikely to be major determinants of treatment decisions, we will not know unless we look.

\section{Disclosures}

The author has no conflicts of interest to declare.

Rami Massie (i) Department of Neurology and Neurosurgery, Montreal Neurological Hospital, McGill University, Montreal, Quebec, Canada

Correspondence to: Rami Massie, Associate Professor, Department of Neurology and Neurosurgery, Montreal Neurological Hospital, McGill University, 3801 University, Montreal, Quebec H3A 2B4, Canada. Email: rami.massie@mcgill.ca

\section{REFERENCES}

1. Koike H, Katsuno M. Transthyretin amyloidosis: update on the clinical spectrum, pathogenesis, and disease-modifying therapies. Neurol Ther. 2020;9(2):317-33.

2. Takei Y, Hattori $\mathrm{T}$, Gono $\mathrm{T}$, et al. Senile systemic amyloidosis presenting as bilateral carpal tunnel syndrome. Amyloid 2002; 9(4):252-5.

3. Gagné F. Intraligamentary amyloid deposits in spinal canal stenosis. Can J Neurol Sci. 1995;22(2):164-7.

4. Pinto MV, Milone M, Mauermann ML, et al. Transthyretin amyloidosis: putting myopathy on the map. Muscle Nerve. 2020; 61(1):95-100.

5. Russel A, Hahn C, Chhibber S, et al. Utility of neuropathy screening for wild-type transthyretin amyloidosis patients. Can J Neurol Sci. 2021.

6. Yungher FW, Kim A, Boehme A, et al. Peripheral neuropathy symptoms in wild type transthyretin amyloidosis. J Peripher Nerv Syst. 2020;25(3):265-72.

7. Fine NM, Davis MK, Anderson K, et al. Canadian Cardiovascular Society/Canadian Heart Failure Society Joint Position Statement on the evaluation and management of patients with cardiac amyloidosis. Can J Cardiol. 2020;36(3): 322-34.

8. Mina C, Bagnato S, Sant'Angelo A, et al. Risk factors associated with peripheral neuropathy in heart failure patients candidates for transplantation. Prog Transplant. 2018;28(1):36-42. 